Instructions for authors, subscriptions and further details:

\title{
Educación y Entorno en la Infancia. Ingredientes Clave en el Desarrollo del Pensamiento Creativo
}

Cecilia Serrano-Martínez ${ }^{1}$

1) Universidad de Zaragoza, España

Date of publication: February $25^{\text {th }}, 2016$

Edition period: February 2016-June 2016

To cite this article: Serrano, C. (2016). Educación y Entorno en la Infancia. Ingredientes Clave en el Desarrollo del Pensamiento Creativo. International Journal of Sociology of Education, 5(1), 67-84. doi: 10.17583/rise.2016.1896

To link this article: http://dx.doi.org/10.17583/rise.2016.1896

PLEASE SCROLL DOWN FOR ARTICLE

The terms and conditions of use are related to the Open Journal System and to Creative Commons Attribution License (CC-BY) 


\section{Education and Environment in Childhood. Key Ingredients in the Development of Creative Thinking}

Cecilia Serrano-Martínez

Universidad de Zaragoza

(Received: 18 January 2016; Accepted: 5 February 2016; Published: 25

February 2016)

\section{Abstract}

Education and the environment experienced in childhood help or hinder the development of creative thinking. The various creative experiences that occur in childhood and the context in which these experiences are developed are key in choosing a profession and/or training pathway in adulthood. This is the case of different professionals whose decision to choose a trade was marked during their educational period. We have been eight life stories of creative workers in the region of Aragon (Spain), considering creative jobs list offered by Florida (2002). Some of the conclusions are the importance of the development of critical and creative thinking in the classroom and their connexion with family, friends, institutions, etc. The environment is a key aspect to understand the creative development of these professionals.

Keywords: education, environment, creativity, family, educational dialogue. 


\section{Educación y Entorno en la Infancia. Ingredientes Clave en el Desarrollo del Pensamiento Creativo}

Cecilia Serrano-Martínez

Universidad de Zaragoza

(Recibido: 18 de Enero 2016; Aceptado: 5 de Febrero 2016; Publicado: 25 Febrero 2016)

\section{Resumen}

La educación y el entorno vivido en la infancia favorecen o dificultan el desarrollo de un pensamiento creativo. Las diversas experiencias creativas que acontecen en la infancia y el contexto en el que se desarrollan son claves en la elección de una profesión y/o un itinerario formativo en la edad adulta. Este es el caso de diferentes profesionales cuya decisión de escoger un oficio u otro, estuvo marcada durante su periodo educativo. Hemos realizado ocho historias de vida a trabajadores creativos de la región de Aragón (España), considerando como empleos creativos el listado realizado por Florida (2002). Algunas de las conclusiones son la importancia del desarrollo de un pensamiento crítico y creativo en las aulas y su conexión con la familia, amigos, instituciones, etc. El entorno es un aspecto clave para entender el desarrollo creativo de estos profesionales.

Palabras clave: educación, entorno, creatividad, familia, diálogo educativo. 


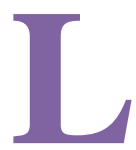

a educación y el entorno vivido en la infancia favorecen o dificultan el desarrollo de un pensamiento creativo. Las diversas experiencias creativas que acontecen en la infancia y el contexto en el que se desarrollan son claves en la elección de una profesión y/o un itinerario formativo en la edad adulta. Este es el caso de diferentes profesionales cuya decisión de escoger un oficio u otro, estuvo marcada durante su periodo educativo. Hemos realizado ocho historias de vida a trabajadores creativos de la región de Aragón (España), considerando como empleos creativos el listado realizado por Florida (2002). Concretamente, nos hemos centrado en el núcleo denominado como "súper creativo" por el citado autor. Éste consiste en una

"nueva clase de científicos, ingenieros, profesores universitarios,
poetas, actores, novelistas, animadores, artistas, arquitectos y
diseñadores, trabajadores culturales, pensadores científicos,
analistas y formadores de opinión, cuya función económica es
crear nuevas ideas, nueva tecnología y/o un nuevo contenido
creativo" (Florida, 2002, p. 8).

En primer lugar, para hablar de educación hay que tratar en paralelo a la familia, ya que la selección de la educación en los niños está seleccionada en gran parte por los progenitores. En segundo término, habría que valorar el entorno en el que se vive y las opciones existentes en la elección de un colegio u otro. En este sentido, Milgram (1990) señala que el colegio, el hogar y la comunidad, influyen en el desarrollo de diferentes potencialidades y habilidades. En cuanto al entorno escolar, la autora considera que todo el tiempo que el niño está en el colegio y las experiencias que en el mismo experimenta, contribuirán al desarrollo de sus habilidades. El entorno familiar provee al menor la calidad y la cantidad de comportamientos y actitudes que, por su interacción con los mismos, pueden crear ciertos intereses en los niños y servir de influencia para el desarrollo de sus habilidades intelectuales. El entorno comunitario hace referencia a que las comunidades influyen en las oportunidades específicas de la educación especial que está a disposición de los estudiantes (decisiones políticas, experiencias educacionales, defensa de la educación, etc.).

Lo anterior tiene que ver con la teoría de Mead (Joas, 2005) en cuanto a que toda su estrategia conceptual se dirige hacia un concepto no 


\section{Serrano-Martínez-Pensamiento Creativo}

individualista de la acción social. Este autor entiende que el "acto social" se refiere a diferentes actos individuales que tienen lugar como un grupo complejo de actividad. La teoría de la educación de Mead, según Joas (2005, p. 138), no era precisamente una pedagogía de la adaptación. Se trataba más bien el intento de permitir que el niño crezca en situaciones problemáticas en las que adquiera un fuerte interés que le impulse a formase una conducta basada en su propia resolución creativa de problemas.

El valor del entorno como influencia en el individuo también ha sido estudiado por McCleland (1961, p. 373), que indica que existen influencias indirectas que afectan a los niveles de logro. Distingue entre cuatro principales variables: el contexto físico, la familia, la economía y el entorno. El desarrollo de las personas emprendedoras en una sociedad depende, en parte, de las prácticas de socialización temprana y crianza de los hijos, llevadas a cabo en la sociedad de la que forman parte.

Una fuente fundamental en la creación de una identidad y de una educación es la generada a través de las instituciones educativas. En relación con esta idea, Parsons (1990) considera que en la escuela se produce una especie de "resocialización", en la que el niño obtiene una nueva identificación alejada de su identidad de origen. Esto ocurre al aplicar el modelo educativo de igualdad de oportunidades, el cual elimina las posibles diferencias sociales existentes entre los compañeros. Opina que en el colegio se cumplen dos metas o misiones: lograr que los alumnos asimilen una serie de conocimientos y destrezas (aprendizaje cognitivo), e infundir una "orientación moral".

Para Bourdieu (1984), uno de los fundamentos del capital educativo es su propensión a cuestionar un orden social, el cual no reconoce plenamente sus méritos. Se trata de un orden que tiene en cuenta otros principios de clasificación, que no son aquellos que el propio sistema educativo ha utilizado para clasificarlos. La función del sistema educativo es "transformar los talentos de los individuos en las competencias y habilidades necesarias para desempeñar las ocupaciones más importantes" (Requena y Bernardi, 2008, p. 244). Otro ejemplo de los beneficios aportados por las instituciones educativas es la teoría de la acción colectiva, por la que Joas (2005) sitúa a la escuela como espacio para la movilización de recursos, ya que son las propias instituciones las que se consideran como actores racionales. El citado autor indica que la participación en el acto colectivo por cada 
individuo tiene numerosas ventajas. En relación a la sobrevaloración realizada a las instituciones, Castoriadis (1983, p. 201) considera que "las instituciones no se reducen a lo simbólico, pero no pueden existir más que en lo simbólico, son imposible fuera de un simbólico en segundo grado y constituyen cada una su red simbólica".

Una manera más global de entender la educación es a través de las aportaciones de Feinstein, et al. (2008), según el cual, la educación tiene que ver con el contexto, proceso y aprendizaje. El sentido otorgado al proceso se centra en aquellos contextos de aprendizaje en el que se desarrollan y experimentan diferentes tipos de aprendizajes dirigidos. Los citados autores indican que el significado de la experiencia incluye la interacción con el profesor a través de diferentes etapas educacionales cuyo objetivo es el desarrollo de una serie de competencias clave, conocimientos, valores y capacidades.

\section{Metodología y Objetivos}

El presente artículo tiene como objetivo conocer las experiencias educativas en la infancia de aquellos profesionales considerados como creativos. Concretamente, nos interesa la parte bibliográfica relacionada con la educación y la familia, como elementos decisorios de una u otra profesión en la edad adulta. Trataremos de comprender los factores asociados a la educación en la infancia y su relación con el pensamiento creativo.

La metodología está basada en la realización de ocho historias de vida. Se tratan de relatos autobiográficos que obtiene el investigador de los entrevistados. La información es extraída a partir de las experiencias narradas en diversas entrevistas, las cuales se realizan sucesivamente. El objetivo de esta técnica, según la definición de Pujadas (2002, p. 48), es "mostrar el testimonio subjetivo de una persona en la que recojan tanto los acontecimientos como las valoraciones que dicha persona hace de su propia existencia".

La metodología se basa en una muestra de tipo estructural. Los perfiles entrevistados pertenecen al territorio aragonés y se han seleccionado en función de las regiones en las que hay mayor representación de creativos por provincia. Otros de los criterios muéstrales son: la ocupación, el ranking de creatividad comarcal $^{1}$, la edad y el género. Para esta selección nos hemos 


\section{Serrano-Martínez-Pensamiento Creativo}

ayudado de la proporción de ocupaciones creativas por comarcas obtenidas a partir del Censo del 2001. Los perfiles seleccionados son los siguientes:

Perfil 1: Profesionales en organizaciones de empresas, profesionales en las ciencias sociales y humanas asociadas a titulaciones de $2^{\circ}$ y 3 er ciclo universitario (Economistas, Sociólogos, historiadores, filósofos, filólogos, psicólogos y asimilados, etc.). Mujer, menor de 40 años, D.C. Zaragoza, (Zaragoza, Aragón) Empresaria-Historiadora. (En adelante HV1)

Perfil 2: Profesiones asociadas a una titulación de 1er ciclo universitario en ciencias físicas, químicas, matemáticas, ingeniería y asimilados (Arquitectos técnicos, informáticos, ingenieros). Mujer, mayor de 40 años, D.C. Zaragoza, (Zaragoza, Aragón). Arquitecta. (En adelante HV2) Perfil 3: Profesiones asociadas a titulaciones de $2^{\circ}$ y $3 \mathrm{er}$ ciclo universitario en ciencias físicas, químicas, matemáticas e ingeniería. (Matemáticos, Físicos, químicos, ingenieros superiores, arquitectos superiores, etc.) Varón, menor de 40 años, D.C. Zaragoza (Zaragoza, Aragón). Científico. Ingeniero químico. (En adelante HV3)

Perfil 4: Escritores, artistas y otras. Varón, mayor de 40 años, Hoya de Huesca (Huesca, Aragón). Ilustrador, diseñador. (En adelante HV4)

Perfil 5: Profesiones asociadas a titulaciones de $2^{\circ}$ o $3 \mathrm{er}$ ciclo universitario en ciencias naturales y sanidad (médicos, veterinarios, farmacéuticos). Varón, mayor de 40 años, Bajo Aragón (Teruel, Aragón). Médico de familia. (En adelante HV5)

Perfil 6: Profesiones asociadas a titulaciones de $2^{\circ}$ o $3 e r$ ciclo universitario en la enseñanza (Profesores de Secundaria y de Enseñanza Universitaria). Mujer, menor de 40 años, La Jacetania (Huesca, Aragón). Profesora de instituto. (En adelante HV6)

Perfil 7: Dirección de empresas de 10 a más asalariados (Dirección de áreas y departamentos especializados, etc.) Varón, menor de 40 años, Campo de Daroca (Zaragoza, Aragón). Gerente de empresa, biólogo y comercial. (En adelante HV7)

Perfil 8: Profesionales del derecho (abogados, fiscales, jueces, magistrados) Mujer, menor de 40 años, Comunidad de Teruel (Teruel, Aragón). Abogada. (En adelante HV8) 
En definitiva, se han realizado ocho historias de vida distribuidas paritariamente del siguiente modo: dos varones menores de 40 años, dos varones mayores de 40 años, tres mujeres menores de 40 años y una mujer mayor de 40 años. En el siguiente análisis denominaremos los perfiles siguiendo el listado anterior, tal y como resumimos en el siguiente cuadro.

Tabla 1

Denominación de los perfiles en el análisis

\begin{tabular}{lcc}
\hline \multicolumn{1}{c}{ Profesión } & $\begin{array}{c}\text { Núm. de } \\
\text { perfil }\end{array}$ & $\begin{array}{c}\text { Modo de } \\
\text { denominarlo }\end{array}$ \\
\hline Empresaria-Historiadora & 1 & HV1 \\
Arquitecta & 2 & HV2 \\
Científico. Ingeniero químico & 3 & HV3 \\
Ilustrador, diseñador & 4 & HV4 \\
Médico de familia & 5 & HV5 \\
Profesora de instituto, filóloga & 6 & HV6 \\
Gerente de empresa, biólogo y comercial & 7 & HV7 \\
Abogada & 8 & HV8 \\
\hline
\end{tabular}

Fuente: Elaboración propia, según los perfiles entrevistados

\section{Discursos de los Profesionales Creativos}

A lo largo de la investigación cualitativa contrastaremos los discursos de los profesionales creativos seleccionados. Uno de los temas que más aparecen al compartir sus experiencias educativas son las influencias que experimentaron durante su infancia en su entorno familiar. Se tratan de elementos contextuales que tuvieron cierto peso en la elección de sus 


\section{Serrano-Martínez-Pensamiento Creativo}

estudios, como fuerza motivadora e impulso para tomar decisiones hacia una rama o disciplina académico-profesional. Del mismo modo, comparten sus sensaciones en torno a la autoridad y el diálogo que tenían con sus profesores. Esto se debe a que la figura del profesor es un elemento clave en las biografías de estos individuos. En esto último hay tres discursos diferenciados, según el momento histórico en el que asistieron a cursos de COU o BUP; dependiendo como se denominase en función de la ley educativa del momento. Al margen de cómo fuese, se refieren fundamentalmente a los cursos de los que tienen más recuerdos, que corresponden a aquellos en los que tenían entre 12 y 18 años. Estos tres bloques de discursos más reiterados se centran en: educación e influencias, autoridad y diálogo educativo, y estudios, premios y becas.

\section{Educación e Influencias}

La decisión de qué estudios cursar es escogida por los progenitores durante sus primeros cursos escolares, ya que seleccionan el colegio al que inscribirlos, lo cual les puede condicionar hacia un determinado pensamiento $^{2}$. La influencia en la educación temprana es directa, o más bien, no se trata de una influencia sino de una decisión tomada desde el seno familiar; lo cual está relacionado con los valores que los padres quieren que sus hijos alcancen en el sistema educativo. El menor se identifica con estos significantes ofrecidos por sus padres y madres y los interioriza, es decir, el niño/a "acepta los roles y las actitudes de los otros significantes, o sea que los internaliza y se apropia de ellos” (Berger y Luckmann, 2006, p. 165). A partir de los reflejos externos anteriores, el menor es capaz de identificarse él mismo y de adquirir una identidad, la cual es coherente con todo lo que ha experimentado e interiorizado.

En cuanto al rol que tienen los padres en el logro educacional de sus hijos, Feinstein, et al. (2008, p. 24) indican que existe una transmisión intergeneracional centrada en el éxito educativo. Del mismo modo, por medio de su modelo ecológico, explican que la clave en la infancia incluye a la familia, el entorno preescolar, los vecinos y los grupos de pares. Incluyen un modelo conceptual que muestra los efectos que tiene la educación que ejercen los padres a los hijos en el rendimiento escolar. Por ello, las influencias de sus progenitores no solo tienen que ver con la elección del 
centro de estudios, sino que hay que valorar el papel educacional que sus padres ejercieron durante su infancia. En este sentido, el mapa conceptual de los citados autores (p. 26) puede resultarnos de utilidad para mostrar gráficamente todas influencias relacionadas con la educación y la infancia.

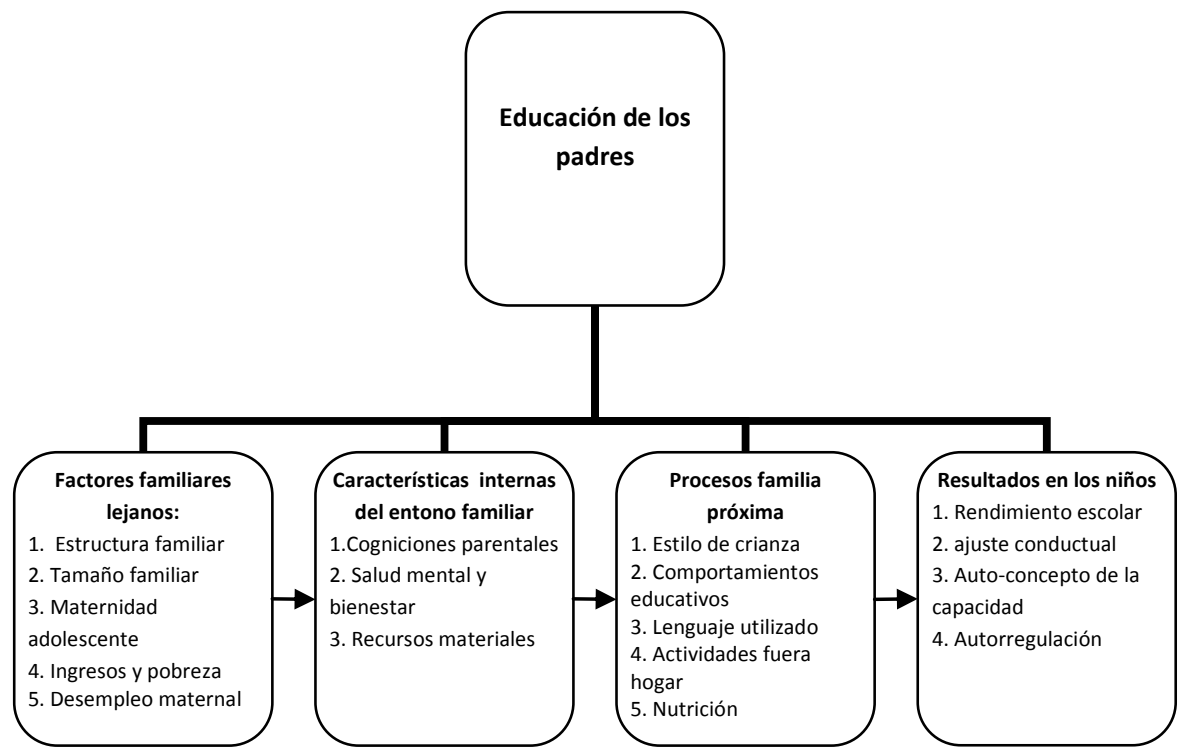

Figura 1. Modelo conceptual de los efectos de la educación de los padres en los logros de sus hijos en el colegio

Fuente: Adaptación propia de la figura aportada por Feinstein, et al. (2008, p. 26)

La figura anterior presenta una serie de situaciones o aspectos de la educación de los padres que pueden repercutir, directa o indirectamente, en la manera en la que sus hijos se desarrollan en su entorno distante y próximo. No obstante, a partir de la adolescencia, la influencia del medio cercano y familiar empieza a ser menos intensa. A partir de este periodo vital comienzan a tener más importancia el grupo de pares, es decir, su grupo de amigos o los compañeros de clase. Un ejemplo de esta desvinculación del seno familiar es que, a partir de la adolescencia comienza a tener más peso su opinión propia en la elección de su itinerario escolar. En cuanto a estudios 


\section{Serrano-Martínez-Pensamiento Creativo}

superiores, ya sea Formación Profesional o estudios universitarios, no hay ningún patrón común relacionado con las posibles influencias que tuvieron sus padres en la elección de sus estudios superiores. Algunos de ellos, desde edades muy tempranas, ya sentían una identificación con un tipo de carrera universitaria determinada, como en el caso de la arquitecta. Desde el punto de vista de Winnicott (1971, p. 150), los adolescentes realizan un esfuerzo y una confrontación personal por medio de diversos actos. Añade que, en el caso de que las figuras parentales abdicasen de su responsabilidad en el crecimiento de sus hijos, los adolescentes perderían su activo más importante, que es "la libertad de tener ideas y actuar por impulso". En el otro lado, aparecen discursos que indican que su interés por una rama determinada surgió a partir de la influencia positiva de un profesor en concreto; por el hecho de que impartía bien una asignatura, o por la facilidad de los contenidos existentes en la misma. Las aficiones que tenían desde pequeños en su familia, como la lectura o el arte, determinan la elección de los estudios en el caso de la historiadora y la filóloga. Dejarse llevar por el azar o el instinto casi determina los estudios del químico. Como podemos observar, las pautas en la elección de los estudios que deciden cursar están fundamentalmente marcadas por criterios intrínsecos (motivación personal, sensaciones) y extrínsecos (aficiones aprendidas, admiración a un profesor).

En cuanto a los intereses de los padres en el cuidado y educación de los hijos, Coleman (2011, p. 739) señala que existen tres dimensiones en las que se pueden observar los conflictos potenciales que se generan entre la sociedad y la familia. El primero de ellos es la confrontación existente entre "los valores, orientaciones costumbres, lengua, normas y cultura" de la familia frente al orden social, por lo que se trata de un conflicto cultural. En segundo lugar tenemos un conflicto educativo ya que, por un lado, la familia está interesada en usar sus propios recursos para educar sus hijos y, por otro lado, la sociedad busca servirse de los recursos del conjunto de las familias. En tercer lugar aparece el conflicto generacional, puesto que los padres presentan interés en invertir sus recursos en sí mismos, pero el orden social trata de gastar sus recursos para la próxima generación.

La relación entre padres y profesores varía en función de la época en la que el entrevistado era estudiante y de la filosofía propia de la institución educativa. También estaba muy influenciado por el perfil profesional de los padres, ya que aquellos que eran educadores estaban más atentos al 
desarrollo del menor, tal y como indican en una ocasión:

"Mi padre era profesor y entonces lo que yo sí que recuerdo es que llegaba, me sacaba todos libros de la cartera, me revisaba todos los deberes que había hecho, me pedía todo lo que tenía que hacer" (HV3).

El ejemplo anterior no es un fenómeno aislado, ya que aquellos que tenían uno de los padres profesores, coinciden en esta apreciación. En los años de la dictadura franquista los padres otorgaban una confianza plena al profesor, tal y como señalan:

"El primer año de estar en Zaragoza, con doce años, mi madre le dice al cura: -este niño es muy movido, si tiene que pegarle, péguele-. Y yo eso lo tengo en la cabeza porque también recibí buenas collejas de los curas, por supuesto. Pero era la forma de actuar, o sea, yo no se lo reprocho a mi madre" (HV4).

Hay casos en los que los padres tenían un contacto directo y continuado con el profesorado e intentaban asistir a todas las reuniones, y otros en los no mantuvieron diálogos de forma asidua. En ambos dos, los creativos preferían que no hubiese mucho contacto para no permanecer bajo ese control constante. En el otro margen aparecen visiones en las que los padres no se vinculaban con el colegio ni con el profesorado. En estos casos, el sentimiento generado era de una especie de abandono o de falta de interés hacia el progreso del menor.

"Yo era la última y pasaban de mi como la mierda, tía. Era alucinante, o sea, es que yo recuerdo mi fiesta de marianistas, que aunque ya íbamos a segundo o a tercero de BUP hicimos un baile, y fui la única, (...), de mí pasaron como de la mierda y no me fueron a ver. Yo eso lo llevaba como mal, que me encantaba porque era como la típica guay que mis padres no iban a las reuniones. Pasaban mucho..." (HV1).

El ejemplo anterior muestra un desapego fraternal hacia las actividades educativas en las que tenían cabida la familia. Esta variedad de modos de establecer el contacto entre el contexto educativo y el familiar terminaba por 


\section{Serrano-Martínez-Pensamiento Creativo}

crear una autopercepción de su función como estudiante, según la cual, se desarrollaban una serie de valores otorgados al estudio en la que los padres poseían una influencia considerable.

\section{Autoridad y Diálogo Educativo}

Hay diferentes estudios que se centran en la interacción del alumno con el profesor y en el clima en el aula. Una relación positiva entre el profesor y el alumno es importante para que los estudiantes alcancen mejores logros y mayores compromisos en el aula (Roorda, et al. 2011, citado en Van Uden, et al. 2014, p. 22). En cuanto a la atención recibida por parte del profesorado, Feinstein, et al. (2008, p. 128), indican que las desigualdades en el aula se explican como un producto de las actitudes que los profesores mantienen hacia las expectativas de aquellos alumnos procedentes de entornos socioeconómicos más bajos. En los casos estudiados, las actitudes del profesorado varían dependiendo de la época en la que cursaron sus estudios. Del mismo modo, en función del perfil del profesorado se fomentaba en el alumnado unas capacidades u otras. Estas diferencias dependen en gran medida del momento histórico en el que estudiaron los entrevistados. Podemos diferenciar entre tres grupos de experiencias en los estudios pre-universitarios: principios de los años 70, finales de la década del 70 y principios de los años 80 , y finales de los 80 hasta mediados de la década del 90. En primer lugar, en el caso de los que realizaron el COU a principios de los 70, indican que: "la distancia con los profesores en aquella época era siempre muy importante" (HV5). La mayoría de esta generación asistió a colegios religiosos, por lo que estaban inmersos en un sistema normativo que marcaba su comportamiento. De nuevo aparece el asunto de la autoridad, aspecto clave para comprender parte de la socialización familiar y educativa en la que estuvieron inmersos.

En segundo lugar, un tercio de ellos pertenecen a una generación que asistieron al instituto en un contexto socio-político en el que España acababa de dar los coletazos a una dictadura militar (finales de los 70`s). En general, se trata de un profesorado que apostaba por aplicar nuevas metodologías educativas.

"Yo tengo profesores en el instituto que hacen apuestas importantes por valores y por formas de enseñanza diferentes y que van mucho 
más allá de la enseñanza y se sitúan en un terreno de la pedagogía muy interesante. $\mathrm{O}$ sea, los valores que nosotros hemos adquirido en esa enseñanza han sido valores muy positivos, verdaderamente interesantes. Esos profesores son los primeros que ponen en marcha una forma de enseñanza nueva" (HV4).

Parte de este pensamiento progresista consistía en tener en cuenta la opinión de los estudiantes para tomar aquellas decisiones que interesaban a la comunidad educativa. Realizaban asambleas y otras actividades caracterizadas por fomentar el componente participativo en el alumnado, como la participación en revistas de opinión. Estos profesores les otorgaban parte de la responsabilidad de su propia educación. Esta libertad de acción les convertía en autónomos desde edades tempranas y les ofrecía "un territorio muy amplio de posibilidades" (HV4); siendo ellos mismos los que autorregulaban sus decisiones. Estas actitudes innovadoras de los profesores hacia los alumnos favorecen el logro y el éxito del alumnado, y del mismo modo reduce la percepción acerca de la dificultad (Van Uden, et al. 2014, p. 23).

En último término, los entrevistados no recuerdan que existiesen normas muy estrictas en sus colegios y tienen recuerdos positivos. No obstante, aquellos que tuvieron una educación puramente cristiana no mantenían una relación muy estrecha con el profesorado. Uno de los problemas clásicos de los colegios, tal y como señala Rickards (1988, p. 12), es que se continúa asumiendo que muchos de los problemas tienen una sola respuesta correcta. Esta creencia bloquea las oportunidades de desarrollar la creatividad y la imaginación. El citado autor considera que esta determinación es un error, ya que los problemas de una sola respuesta se encuentran con menos frecuencia en las personas imaginativas, puesto que se caracterizan por soluciones definidas de manera inequívoca y estable en el tiempo.

En tercer lugar, la generación de creativos más jóvenes, que son los terminaron sus estudios pre-universitarios a comienzos de la década del 90 , no presentan una figura del profesorado común. Se daban casos de profesores estrictos y de docentes con los que tenían mayor cercanía y confianza. Los recuerdos de profesores que han permanecido en su memoria están determinados por la capacidad de aprendizaje que adquirieron de los mismos, independientemente de la intensidad emocional que mantuvieron. 


\section{Serrano-Martínez-Pensamiento Creativo}

"Era un tío de ultraderechas, pero le encantaba la biología y nos transmitía. Puede que sea el profesor más estricto de los que he tenido en mi vida y es el único de los que me acuerdo de su nombre y me acuerdo de su cara. Era estricto pero era justo, y nos transmitió mucho amor hacía la biología. Yo, por su culpa o gracias a él, estudié biología" (HV7).

Cabe indicar que, aquellos que tenían una relación de confianza con los profesores, han mantenido el contacto con ellos con el paso de los años. Este contacto ha estado motivado, bien por una vinculación puramente profesional (pedir consejo, trabajo, participación en proyectos, etc.), o por amistad.

\section{Estudios, Premios y Becas}

Al hablar de estudios aparecen diferentes matizaciones referidas al grado realizado, como son el contexto educativo de la infancia, los estudios preuniversitarios y la carrera universitaria o las especializaciones en una disciplina concreta. Muchos de los creativos tuvieron una educación cristiana durante sus primeros años de escolarización. El colegio era una elección familiar, por lo que el centro era impuesto por los padres sin contar con la opinión del menor. A partir de la adolescencia o de la mayoría de edad es el momento en el que muchos de los creativos cambian la educación cristiana por la educación aconfesional y pública.

La mayor parte de los entrevistados ha desarrollado otras de sus habilidades fuera del contexto educativo instituido, es decir, han alcanzado otros títulos no universitarios que les han servido para trabajar durante un periodo de sus vidas y para enriquecerse personalmente. Del mismo modo, han tenido que viajar a otras ciudades o países (Francia, Pamplona, Madrid, etc.) para continuar formándose, ya que en Aragón no existían los estudios o cursos que deseaban realizar. Normalmente en estos viajes han conocido a personas afines a su perfil profesional, con las que han mantenido el contacto o han realizado proyectos en ocasiones puntuales.

Una variable común en los entrevistados es que, en alguna ocasión, recibieron premios o becas por sus méritos; ya sea por sus buenas notas, habilidades plásticas o literarias, así como por participar en concursos del colegio o de su ciudad, etc. Alguno de ellos recibió otras becas que no 
estaban necesariamente relacionadas con el esfuerzo propio, sino que se otorgaban por factores objetivos, como los de ser familia numerosa o por los ingresos económicos de los padres. En su edad adulta alguno de los entrevistados ha recibido distinciones y premios relacionados con su actividad laboral o con proyectos concretos e innovadores. No consideran que fueran excesivamente inteligentes (incluso dos de las creativas indican haber suspendido la selectividad), pero sí que le dedicaban tiempo al estudio. La obtención de mejores notas no es un común denominador, pero sí que lo es el interés propio o la exigencia familiar en la consecución de una mejor calificación.

"Siempre he sacado buenas notas y nunca he tenido sensación de esfuerzo para tener que hacerlo. Entonces yo tengo la sensación de que siempre ha sido muy fácil. Por supuesto que le he tenido que dedicar horas, pero ha sido fácil y tampoco he tenido ninguna dificultad para ello" (HV3).

Esta sensación referida a que alcanzaban buenos resultados sin destinar un excesivo esfuerzo, acentúa la idea de que poseían habilidades intrínsecas que no les resultaban excesivamente costosas de desarrollar. Esto también lo señalan en una ocasión en la que ganan un premio de dibujo, diciendo:

"En el instituto hacían un concurso para hacer la portada de la revista, nos obligaron a todos. Yo lo hice, pero vamos, sin currármelo ni nada, y salió elegida la portada" (HV6).

La motivación o el convencimiento en la elección de su itinerario académico es un rasgo común en la mayoría de ellos. El esfuerzo por alcanzar la formación deseada es el impulso principal en la creación de su perfil profesional. Este rasgo está relacionado con la afirmación de Florida (2010, p. 75) referida a que la motivación para la creatividad procede de recompensas intrínsecas, es decir, se trata de una motivación interna. Únicamente aparecen un par de casos en los que escogen una carrera universitaria por descarte, es decir, valorando las opciones disponibles en el territorio aragonés. 


\section{Serrano-Martínez-Pensamiento Creativo}

\section{Conclusiones}

En las siguientes conclusiones sintetizamos aquellos aspectos educativos y familiares que tienen cierto peso en la construcción de los perfiles creativos. Algunos de los puntos fuertes relacionados con la educación de estos profesionales tienen que ver con las influencias que tuvieron durante su temprana infancia, la autoridad, el diálogo educativo y los diferentes premios y becas que obtuvieron o lograron. Las influencias en la educación se ven marcadas por la decisión de sus progenitores durante los primeros años escolares de estos entrevistados. La figura del profesorado genera un mayor o menor apego a las diferentes materias. Aparecen casos de influencias positivas que les llevaron a decantarse por unos estudios o que fortalecieron su espíritu creativo o emprendedor. Lo mismo ocurre con la autoridad, concepto que tiene más peso dependiendo de la época histórica a la que perteneció cada entrevistado. Los cambios sociopolíticos influyen en el pensamiento y en los patrones de liderazgo y comunicación llevados a cabo por los profesores.

El binomio entre autoridad y familia les aportó un gran equilibrio socializador, así como un aprendizaje en la muestra y control de sus emociones. Esta paradoja es clave para entender su modo de ser y la capacidad real de acción y responsabilidad que, posteriormente, han desarrollado en sus vidas personales y profesionales. Los premios y becas que recibieron no tienen demasiada importancia en el posterior desarrollo creativo, siendo las motivaciones intrínsecas las que más efecto tienen en la elección de su profesión o itinerario posterior.

Un elemento clave en la construcción de perfiles creativos es el contexto en el que viven durante su infancia. En todos estos casos, el entorno social de su infancia se caracterizaba por fomentar la imaginación y el aprendizaje resolutivo. Según los resultados alcanzados, es vital valorar la educación recibida en paralelo con la influencia del entorno en el que estos trabajadores se socializaron. En definitiva, las diferentes experiencias que vivieron durante la infancia generan unos aprendizajes que, en el futuro, se transforman en vehículos en la transmisión de unos valores asociados con su identidad profesional. En estos casos, el pensamiento creativo se ha desarrollado en un contexto de diálogo, autoridad e imaginación aplicada. Se tratan de elementos que, pudiendo ser contradictorios entre sí, dotan de 
cierta hibridación que favorece el pensamiento divergente y la vinculación con profesiones caracterizadas por el uso de la creatividad.

\section{Notas}

1 Este análisis corresponde a la parte cuantitativa de la tesis inédita de la autora, titulada: "Sociología de la economía creativa en Aragón. Números, trayectorias vitales y entornos" (Año 2014).

2 También es importante tener presente que no tiene las mismas oportunidades de elección un niño que vive en una zona rural, en la que su colegio esta en otro pueblo al que tiene que desplazarse, que aquel que vive en una gran ciudad con diferentes opciones educativas: colegio público, colegio concertado, colegio privado, etc. Todo ello limita o amplia la capacidad electiva familiar, a la vez que está creando diversas pautas en la biografía del menor.

\section{Referencias}

Berger, P. L., \& Luckmann, T. (2006). La construcción social de la realidad. Buenos Aires: Amorrortu.

Bourdieu, P. (1984). Distinction. London: Routledge.

Castoriadis, C. (1983). La institución imaginaria de la sociedad. Vol. 1 Marxismo y teoría revolucionaria. Barcelona: Ed. Tusquets.

Coleman, J. S. (2011). Fundamentos de teoría social. Madrid: CIS.

Feinstein, L., Duckworth, K., \& Sabates, R. (2008). Education and the

Family. Passing success across the generations. Abingdon: Routledge.

Florida, F. (2002). The rise of the creative class. New York: Basic Books.

Florida, F. (2010) La Clase Creativa. La transformación de la cultura del trabajo y el ocio en el siglo XXI. Barcelona- Buenos Aires. México: Ed Paidós.

Joas, H. (2005). The creativity of action. Cambridge: Polity Press.

McClelland, D. C. (1961). The achieving society. New York: Van Nostrand Company.

Milgram, R. M. (1990). Creativity: An Idea Whose Time Has Come and Gone? En Runco, A., Albert, R. S. (1990). Theories of creativity. California: Sage Publications. 


\section{Serrano-Martínez-Pensamiento Creativo}

Parsons, T. (1990). El aula como sistema social: algunas de sus funciones en la sociedad americana, Educación y Sociedad, 6, 180.

Pujadas Muñoz, J. J. (2002), El método biográfico. El uso de las historias de vida en ciencias sociales. Madrid, CIS (col. Cuadernos metodológicos, $\mathrm{n}^{\mathrm{o}}$ 5).

Requena, M., Bernardi, F. (2008). El sistema educativo en González, J., Requena, M. (2008). Tres décadas de cambio social en España. Madrid: Ed. Alianza.

Rickards, T. (1988). Creativity at work. Aldershot: Gower publishing company.

Serrano-Martínez, C. (2014). Sociología de la economía creativa en Aragón. Números, trayectorias vitales y entornos. (Tesis inédita).

Van Uden, J. M., Ritzen, H., \& Pieters, J. M. (2014). Engaging students: The role of teacher beliefs and interpersonal teacher behavior in fostering student engagement in vocational education. Teaching and Teacher Education, 37, 21-32. doi:10.1016/j.tate.2013.08.005

Winnicott, D. W. (1971). Playing and reality. London: Tavistock Publications.

Cecilia Serrano is Phd Researcher in the Department of Psychology and Sociology at University of Zaragoza, Spain.

Contact Address: Direct correspondence to Cecilia Serrano at Departamento de Sociología, Facultad de Economía y Empresa, Universidad de Zaragoza, Gran Vía, 2 - 50005 Zaragoza, España. E-mail: cserran@unizar.es 